\title{
El efecto del consumo de alcohol sobre el trabajo adolescente en México
}

\section{Effect of alcohol consumption on youth employment in Mexico}

\author{
Juan Francisco Suárez Martínez* \\ Cinthya G. Caamal-Olvera**
}

\begin{abstract}
Resumen
En México, uno de cada cuatro jóvenes de 12 a 17 años consume alcohol y se encuentra trabajando. El consumo de alcohol está asociado a una mayor probabilidad de trabajar a una edad temprana, y la situación laboral podría fomentar un mayor consumo de éste. Para controlar los sesgos por causalidad inversa y por selectividad, se utilizan como instrumentos la influencia de los pares y las emociones de los adolescentes. Los resultados indican una relación directa y robusta entre el consumo de alcohol y el trabajo de los adolescentes, de entre 2.07 y 2.34 puntos porcentuales.
\end{abstract}

Palabras clave: trabajo; jóvenes; alcohol; México.

\begin{abstract}
In Mexico one out of four youths age 12 to 17 years old consume alcohol and are working. It is found that alcohol consumption is associated to a higher probability of working at an early age, and labour participation may increase alcohol consumption. We control for biases due to reverse causality and selectivity by using peer influence and emotions as instruments. We find a direct and robust relationship

* Universidad de Monterrey, Departamento de Economía. Dirección: Ignacio Morones Prieto 4500 Pte., 66238, Monterrey, Nuevo León, México. Correo: juan.suarez@udem.edu ORCID: 0000-0002-6256-0551

** Universidad Autónoma de Nuevo León, Facultad de Economía. Dirección: Lázaro Cárdenas 4600 Ote., Residencial Las Torres, 64930, Monterrey, Nuevo León, México. Correo: cinthya.caamallv@uanl.edu.mx ORCID: https://orcid.org/0000-0003-0249-4027


between alcohol consumption and youth employment in the range of 2.07 and 2.34 percentage points.

Keywords: employment; youth; alcohol; Mexico.

\section{Introducción}

El consumo excesivo de bebidas alcohólicas es un problema de salud pública. Según la OECD (2015), el consumo excesivo de alcohol pasó del octavo al quinto lugar como principal causa de muerte y discapacidad en todo el mundo, entre 1990 y 2010. El abuso de esta sustancia entre los jóvenes tiene un alto costo en la atención médica, los servicios de salud mental, así como el fracaso educativo y los delitos juveniles (Hawkins, Catalano y Miller, 1992).

El consumo de alcohol en los jóvenes afecta su rendimiento intelectual y capacidad de aprendizaje y, por ende, los puede llevar a un bajo rendimiento escolar, que podría ocasionar la deserción escolar. Según cálculos de la Encuesta Nacional de Adicciones (ENA, 2011), se estima que el $84 \%$ de los jóvenes entre 12 y 17 años que asisten a la escuela no consumen alcohol, pero el 16\% restante sí lo hace. El bajo ingreso familiar es una razón para que los jóvenes se inserten al mercado laboral a una edad temprana (Basu y Hoang Van, 1998), pero también los jóvenes decidirían trabajar para financiar el consumo persistente de algún bien, en este caso el alcohol (Mullahy y Sindelar, 1993). La hipótesis que se plantea en este artículo es que, si los jóvenes tienen un consumo excesivo y persistente de alcohol, buscarían formas para financiar su adicción. Si bien existen distintas formas de financiamiento, como estar en actividades ilícitas, robar o adherirse a algún grupo delincuencial o pandilla, también podrían incorporarse en trabajos riesgosos y peligrosos (Keng, 1998). El concepto de trabajo peligroso se refiere al que, por su naturaleza o las circunstancias en las que se lleva a cabo, es probable que dañe o ponga en peligro la salud, la seguridad o la moral de los adolescentes (Guarcello, Lyon y Valdivia, 2016). En México, el $45 \%$ de los jóvenes de 15 a 17 años que trabajan se encuentran en trabajos peligrosos (Guarcello, Lyon y Valdivia, 2016). Por tanto, es de suma importancia investigar las razones que llevan a un adolescente a trabajar en condiciones de riesgo, detectar de forma indirecta los trabajos peligrosos, y buscar soluciones para que esto no ocurra.

La edad es un factor relevante en la asistencia escolar, la inserción al mercado laboral y el inicio en el consumo de alcohol. Aunque los porcentajes de asistencia escolar son similares para mujeres y hombres, la proba- 
bilidad de que los adolescentes asistan a la escuela disminuye con la edad, y la probabilidad de que consuman alcohol se incrementa con la edad. La participación laboral a una edad temprana podría generar impactos negativos en la asistencia escolar y en el logro educativo (Beegle, Dehejia y Gatti, 2009); también afecta la productividad de los individuos en la edad adulta (Forastieri, 2002). Si bien es cierto que algunos tipos de trabajo pudieran influir de manera positiva en los adolescentes, la preocupación se centra en los trabajos peligrosos.

La finalidad de este artículo es analizar si existe una relación entre el consumo de alcohol y el trabajo adolescente (que comprende el grupo de entre 12 y 17 años). El estudio se concentra en México. La estimación empírica impone retos porque podría pensarse que el consumo de alcohol llevaría al adolescente a insertarse al mercado laboral con el fin de financiar su adicción al alcohol, como lo mencionan Mullahy y Sindelar (1993). Por otra parte, si el adolescente trabaja, tendría un ingreso que le permitiría comprar alcohol y, con cierta probabilidad, su consumo podría llegar a ser excesivo, como lo encontraron Selvanathan (1991) y Selvanathan y Selvanathan (2004). En otras palabras, esta relación tiene una causalidad inversa. En esta investigación se utiliza la metodología de variables instrumentales para corregir el problema de endogeneidad por causalidad inversa que presentan las variables relevantes; a su vez, se identifica un sesgo de autoselección porque los jóvenes que consumen persistentemente alcohol y los que se insertan al mercado laboral se comportan de forma similar.

La ENA (2011) permite obtener los datos para estimar la severidad en el consumo de alcohol, que se calcula a partir de la metodología propuesta por la Organización Mundial de la Salud (OMS), a través de una prueba de identificación de los trastornos debidos al consumo de alcohol (AUDIT, por sus siglas en inglés). Para identificar la endogeneidad entre el consumo de alcohol y el trabajo adolescente se utilizan como instrumentos variables relacionadas con el entorno de exposición del adolescente, la influencia de sus pares, así como factores psicológicos y emocionales que lo hacen más propenso a tener un consumo de alcohol peligroso o problemático. Si bien existe una encuesta más reciente, Encodat (2016), sólo se podría incluir un instrumento y de acuerdo con las pruebas de validez de los instrumentos, se encontró que la estimación mejora con la combinación de dos o más instrumentos.

La contribución del artículo consiste en proveer evidencia sobre el efecto del consumo persistente de alcohol sobre la participación laboral de los jóvenes. No existen estudios que aborden la asociación del alcohol y el trabajo adolescente para el caso de México. Los resultados permiten infe- 
rir una asociación directa entre un severo consumo de alcohol y una mayor probabilidad de que el adolescente trabaje; además, esta relación es robusta ante distintas especificaciones tanto de variables de control como de combinaciones de instrumentos.

El diseño de la política pública parece estar enfocado a eliminar el trabajo peligroso, pero no ha considerado a las adicciones como un factor de riesgo para insertarse en este tipo de trabajos riesgosos. Por tanto, para el diseño se requiere contar con información sobre las conductas y factores de riesgo entre los adolescentes, por lo que la recomendación es incluir reactivos que permitan entender las decisiones sobre el consumo de alcohol, así como las habilidades socioemocionales de los jóvenes en el Módulo de Trabajo Infantil (MTI, 2017) de la Encuesta Nacional de Ocupación y Empleo, que es la encuesta que permite identificar el trabajo peligroso.

\section{Revisión de la literatura}

Mullahy y Sindelar (1993), al analizar la relación entre el alcoholismo y el mercado laboral, encuentran que, en el grupo más joven del análisis, dicha adicción tiende a aumentar la participación en el mercado de trabajo y así generar los ingresos monetarios para sufragar tal consumo. También encontraron que los jóvenes con problemas de alcohol son más propensos a tener dificultades en la escuela, aunque podrían combinar la asistencia escolar y el trabajo, o incluso abandonar la escuela. Mullahy y Sindelar (1993) mencionan efectos directos e indirectos del consumo de alcohol sobre la participación laboral, ya que conforme los jóvenes aumentan las horas laboradas acumulan más experiencia, lo que los llevaría a obtener mayores ganancias en el corto plazo; pero en el largo plazo, el logro educativo sería menor que el de los jóvenes que no consumen alcohol de forma frecuente.

Los adolescentes que entran al mercado laboral son más propensos a consumir bebidas alcohólicas, considerando que el tener recursos económicos les permitiría aumentar su consumo, o bien, si preexiste una adicción al alcohol, es probable que busquen participar en el mercado laboral para financiar su adicción (Selvanathan y Selvanathan, 2004; Selvanathan 1991). Por otra parte, Keng y Huffman (2007) no encuentran un efecto significativo entre un mayor ingreso laboral y el consumo intensivo de alcohol. Becker y Murphy (1988) mencionan que el consumo adictivo responde a cambios permanentes en los precios de estos bienes, más que a factores temporales.

Keng (1998) encuentra que, para contener el consumo del alcohol, es más efectivo incrementar un año adicional a la edad legal para consumirlo 
que imponer un impuesto sobre el mismo; mencionan que el abuso del alcohol se ha relacionado con accidentes de tránsito, pérdida de productividad laboral y aumento en los costos de salud. Kandel y Yamaguchi (1987) argumentan que los trabajadores alcohólicos son más propensos a cambiar de trabajo, acumulan menos tiempo en el mismo puesto de trabajo y permanecen más tiempo sin empleo.

Las personas que son adictas al alcohol o a las drogas son más propensas a dejar la escuela y entrar al mercado laboral a una edad temprana (Keng, 1998). Además, si los jóvenes laboran en trabajos peligrosos, tienen más probabilidades de incrementar el consumo de alcohol o de convertirse en bebedores compulsivos (Harris y Fennell, 1988; San José, van de Mheen, van Oers, MackenbacH y Garretsen, 2000). Según datos de ILO (2013), se estima que $13 \%$ de los jóvenes en el mundo se encuentran empleados en trabajos peligrosos.

Los adolescentes se emplean principalmente en trabajos peligrosos (Guarcello, Lyon y Valdivia, 2016), y se ha encontrado que su participación en el mercado laboral tiene efectos negativos en su educación (Ray, 2002; Heady, 2003; Psacharopoulos, 1997; Edmonds, 2007), así como también perciben salarios más bajos en la edad adulta (Ilahi, Orazem y Sedlacek, 2000). Además, las personas que empezaron a trabajar a una edad más temprana autoinforman un peor estado de salud cuando son adultos (Kassouf, McKee y Mossialos, 2001).

Grootaert y Kanbur (1994) muestran que las intervenciones educativas desempeñan un papel clave en la reducción y la eventual abolición del trabajo de los menores de edad. De Janvry, Finan y Sadoulet (2004) indican que los programas de transferencia monetaria condicional, que motivan la asistencia a la escuela, han demostrado ser eficaces para aumentar los logros educativos y reducir el número de trabajadores con minoría de edad. Skoufias, Parker, Behrman y Pessino (2001) encuentran que el Programa de Educación, Salud y Alimentación (Progresa), que consistía en otorgar transferencias condicionadas a los niños para que asistieran a la escuela, indujo aumentos significativos en la asistencia escolar y redujo significativamente la participación laboral de los niños y adolescentes. Schultz (2004) señala que una de cada diez familias beneficiadas por el programa Progresa experimentaron una disminución en las horas laboradas por sus hijos. 


\section{Marco teórico}

En la literatura no se encuentran modelos teóricos que establezcan una relación entre el consumo de bebidas alcohólicas y la decisión de incorporarse al mercado laboral a una edad temprana. Existe más literatura de artículos empíricos que han relacionado el consumo del alcohol con los ingresos laborales obtenidos (Keng, 1998; Keng y Huffman, 2007) y la participación laboral de los jóvenes (Mullahy y Sindelar, 1993; Selvanathan y Selvanathan, 2004; Selvanathan 1991).

La teoría neoclásica proporciona el marco teórico que permite analizar los factores relacionados con la probabilidad de participación laboral. A partir de Cahuc y Zylberberg (2004), aplicamos el modelo para analizar el trabajo adolescente, considerando que el consumo de alcohol es un bien normal, por lo que un mayor ingreso aumentaría su consumo. En este caso, la premisa es que las personas deben estar participando en el mercado laboral para que existan ingresos monetarios positivos en el hogar.

Para facilitar el análisis suponemos como familia representativa a un hogar nuclear, el cual podría estar compuesto por el papá, la mamá y un hijo adolescente. Suponemos que el salario de los padres, $W$, es fijo, pero las horas laborales, $h$, y el ingreso no laboral de los padres, $m$, pueden variar, pues el ingreso del adolescente podría contribuir a incrementar el ingreso familiar o a reducir las horas laborales. El ingreso familiar está compuesto de la siguiente manera:

$$
\text { Ingreso }_{\text {fam }}=\left(h_{\text {papá }}\right) \bar{W}_{\text {papá }}+\left(h_{\text {mamá }}\right) \bar{W}_{\text {mamá }}+m
$$

Basu y Hoang Van (1998) en el axioma de lujo indican que una familia enviará a los hijos a trabajar sólo si el ingreso de los adultos es tan bajo que no les alcanza para comprar la canasta básica y poder subsistir. Por el contrario, los hijos no trabajarían si el ingreso de los padres es mayor o igual al consumo familiar, es decir:

$$
\left(h_{\text {papá }}\right) \bar{W}_{\text {papá }}+\left(h_{\text {mamá }}\right) \bar{W}_{\text {mamá }}+m \geq C_{\text {familiar }}
$$

Representamos el consumo familiar como la suma del consumo de los padres y del adolescente. Suponemos que los padres aportan los recursos para los bienes que consume el adolescente y asumimos el consumo por parte de los padres como fijo. Se podría especificar el consumo de alcohol en cada cesta de consumo, pero en el caso del adolescente no está permitido. Sin embargo, se podría incorporar el consumo de alcohol del adolescente 
en la restricción presupuestaria familiar sólo si el menor logra engañar a sus padres informando un distinto uso del presupuesto asignado al consumo de bienes, suponiendo que el adolescente tiene libertad de elegir alguna parte de su cesta de bienes de consumo.

Con el fin de entender de dónde obtiene el adolescente los ingresos necesarios para financiar el consumo del alcohol, son posibles los siguientes escenarios: 1) que el papá trabaje más horas, para así aumentar su ingreso y poder cubrir el aumento del consumo familiar por parte del adolescente; 2) que la mamá trabaje más horas, para que sea ella quien cubra dicho aumento; 3) ambos padres podrían aumentar las horas laborales y así dividirse el aumento en el consumo del hijo, o bien; 4) el hijo entrará al mercado laboral y será él mismo quien cubra los gastos de su consumo de alcohol.

$$
C_{\text {familiar }}=\bar{C}_{\text {papá }}+\bar{C}_{\text {mamá }}+C_{\text {hijo }}
$$

Para incorporar el consumo del alcohol partimos del modelo de adicción racional propuesto por Becker y Murphy (1988), el cual plantea que la adicción es un comportamiento racional, y que los individuos maximizan su función de utilidad que depende del consumo presente y pasado, considerando que el consumo adictivo es aquel cuyo consumo presente depende del consumo pasado, con lo que se muestra una persistencia en el consumo de alcohol. Los adolescentes son más impacientes que los adultos, y la adicción al consumo de alcohol se incrementa conforme aumenta el grado de complementariedad entre el consumo pasado y presente. Por tanto, podríamos pensar en una situación endógena entre la persistencia en el consumo de alcohol y el ingreso laboral, es decir, esta relación podría ser en ambas direcciones.

No existe consenso si un mayor ingreso está asociado a mayor consumo de alcohol, o si el mayor consumo de alcohol está asociado a un menor o mayor ingreso laboral (Keng, 1998). Por tanto, proponemos analizar la participación laboral de los adolescentes a partir de los resultados de $\mathrm{Mu}-$ llahy y Sindelar (1993), quienes encontraron que el efecto del alcohol es más fuerte sobre la participación laboral que sobre el ingreso laboral. En particular, analizamos los factores asociados con la probabilidad de que un adolescente trabaje, como la persistencia del consumo del alcohol, así como otros factores que también contribuyen en la probabilidad de trabajar, $X$. De esta manera, la ecuación que se desea estimar es la siguiente:

$$
\operatorname{Prob}(\text { Adolescente_trabaja })=f\left(C_{\text {alcohol }}^{*} \mid h_{\text {hijo }}>0, X\right)
$$


Aunque el mecanismo presentado es básico, nos permite establecer una relación directa entre el consumo de alcohol y la participación laboral del adolescente.

\section{Análisis descriptivo}

La información de la ENA (2011) tiene representatividad para la población mexicana de 12 a 65 años, y permite conocer la frecuencia y consumo del alcohol en los últimos doce meses. También proporciona información acerca del estatus laboral en los últimos treinta días, así como datos socioeconómicos individuales y por hogar. Para medir la relación entre la participación laboral de los adolescentes y el consumo de bebidas alcohólicas, el análisis se focaliza entre las personas que tienen entre 12 y 17 años.

La participación laboral adolescente se mide de forma dicotómica, ya que toma el valor de uno si el joven respondió que trabajó, y de cero si no lo hizo. Con el fin de comparar la situación de los adolescentes que trabajan y los que no lo hacen, en el Cuadro 1 se presenta información descriptiva que muestra que una mayor proporción de hombres trabajan (75.53\%), en comparación con las mujeres (24.47\%). Una mayor proporción de los adolescentes de 15 a 17 años trabajan (71.26\%), mientras que en el grupo que no tiene la edad mínima para laborar, de 12 a 14 años, sólo el 28.73\% trabaja. La gran mayoría de los adolescentes que no trabajan mencionan que asisten a la escuela (88.06\%), y poco más de la mitad de los que trabajan van a la escuela $(54.19 \%)$. No se encuentran diferencias significativas en la composición del hogar nuclear, pero sí en el tamaño del hogar de los jóvenes según su estatus laboral. Entre regiones existen diferencias entre el porcentaje de jóvenes que trabajan y los que no lo hacen: en la regiones norte y centro hay una mayor proporción de jóvenes que no trabajan (32.59 y 40.12\%, respectivamente), mientras que en las regiones centro-norte y sur es en donde hay una mayor proporción que trabaja.

Para estimar la persistencia en el consumo de alcohol se debe considerar no sólo si el joven lo ha probado, sino también la cantidad y la frecuencia de su consumo. Por esta razón utilizamos la prueba de identificación de los trastornos debidos al consumo de alcohol (AUDIT, por sus siglas en inglés), que fue desarrollado por la Organización Mundial de la Salud (OMS) con el fin de detectar el consumo excesivo de alcohol, así como una posible dependencia. El cuestionario del AUDIT es una prueba de escrutinio con estándar internacional que consta de 10 preguntas, mismas que se encuentran en el cuestionario de la ENA (2011). Cada pregunta mide la severidad en 
el consumo, tomando un valor de cero a cuatro, de tal forma que, mientras más consume y con mayor frecuencia, obtiene un mayor valor numérico. Al finalizar la prueba, se suman los valores obtenidos de cada pregunta, y dependiendo de este puntaje es el tipo de consumo que tiene cada individuo. El puntaje final del AUDIT se encuentra en el intervalo [0.40], y mientras mayor sea el valor de AUDIT, la persona se encuentra en un consumo de alcohol más peligroso. Una explicación más detallada se encuentra en Babor, Higgins-Biddle, Saunders y Monteiro (2001) y Reinert y Allen (2002). En el caso de México, Telumbre-Terrero y Sánchez-Jaimes (2015) y Guzmán, Pedrão, Rodríguez, López y Esparza (2007) han aplicado la prueba AUDIT para medir la frecuencia y la severidad del consumo de alcohol.

Se encuentra que la mayor parte de los adolescentes están en la clasificación de no consumo o de bajo riesgo (95.47\%), mientras que el 3.43\% aparece en consumo riesgoso, $0.50 \%$ se ubica en peligroso, y $0.60 \%$ en consumo problemático. El Cuadro 1 muestra la comparación de la severidad en el consumo de alcohol y la situación laboral. La mayor parte de los adolescentes que no reportan consumo de alcohol no trabajan (74.81\%) y tienen un valor del AUDIT de cero; una situación similar se observa con los adolescentes que no consumen alcohol y trabajan (58.46\%). Las diferencias entre el consumo de alcohol y la participación laboral se encuentran en los porcentajes de adolescentes que reportan consumo en riesgo, peligroso y problemático, ya que se estiman mayores porcentajes de jóvenes que trabajan en comparación con los que no lo hacen: las diferencias son estadísticamente significativas con excepción del consumo peligroso. Aunque los porcentajes de consumo peligroso y problemático resultan ser valores muy pequeños, la severidad de este consumo preocupa, pues son jóvenes que reportaron que consumen alcohol casi a diario, toman al menos siete bebidas, son incapaces de detener el consumo una vez que inician, han necesitado beber alcohol en ayunas para recuperarse del día anterior, no recuerdan lo que pasó la noche anterior, o bien, algún familiar se ha preocupado por la intensidad de su consumo alcohólico.

\section{Estrategia empírica}

La estrategia empírica consiste en examinar el efecto del consumo de alcohol sobre la participación laboral de los adolescentes. La intuición de analizar las variables en esta dirección responde a que los adolescentes, con un determinado nivel de consumo de alcohol, buscarán trabajar para obtener ingresos que financien su adicción. Como resultado, la probabilidad 
de trabajar aumentaría, aunque sus opciones serán limitadas debido a que no tienen la edad legal para hacerlo, y probablemente padezcan una severa adicción. Si bien no es posible identificar los trabajos de explotación o los peligrosos, los adolescentes estarían propensos a encontrar algún trabajo que no cumpla con las normas de seguridad, o que no tenga las condiciones laborales mínimas según la Ley Federal del Trabajo.

\section{Cuadro 1}

Estadísticas descriptivas adolescentes de 12 a 17 años

\begin{tabular}{lccc}
\hline & Trabaja & No trabaja & $\begin{array}{c}\text { Prueba } \\
\text { de diferencias }\end{array}$ \\
\hline Hombres (\%) & $75.53 \%$ & $45.71 \%$ & $-13.83^{* * *}$ \\
Mujeres (\%) & $24.47 \%$ & $54.29 \%$ & $13.83 * * *$ \\
Menores de 12 a 14 (\%) & $28.73 \%$ & $54.81 \%$ & $12.03 * * *$ \\
Menores de 15 a 17 (\%) & $71.26 \%$ & $45.18 \%$ & $-12.03 * * *$ \\
Asistencia escolar (\%) & $54.19 \%$ & $88.06 \%$ & $21.44 * * *$ \\
Hogares nucleares (\%) & $80.53 \%$ & $78.75 \%$ & -0.9864 \\
Seguro médico & $70.74 \%$ & $77.76 \%$ & $3.73 * * *$ \\
(público o privado) (\%) & & & \\
Habitantes del hogar & 5.44 & 5.02 & $-2.06 * *$ \\
\hline Regiones & & & \\
Norte & $24.30 \%$ & $32.59 \%$ & $4.05 * * *$ \\
Centro-norte & $30.38 \%$ & $25.00 \%$ & $-2.78 * * *$ \\
Centro & $15.76 \%$ & $40.12 \%$ & $1.88^{*}$ \\
Sur & $29.06 \%$ & $23.20 \%$ & $-1.70 *$ \\
\hline Clasificación AUDIT-OMS & & & \\
Sin consumo (0) & $58.46 \%$ & $74.81 \%$ & $8.34 * * *$ \\
Bajo riesgo (1-7) & $33.00 \%$ & $22.80 \%$ & $-5.71 * * *$ \\
En riesgo (8-15) & $6.73 \%$ & $2.10 \%$ & $-6.35 * * *$ \\
Peligroso (16-19) & $0.66 \%$ & $0.37 \%$ & -1.007 \\
Problemático ( $\geq 20)$ & $1.15 \%$ & $0.43 \%$ & $-2.21 * *$ \\
\hline Observaciones & 609 & 3240 & \\
\hline & & & \\
\hline & & & \\
\hline
\end{tabular}

${ }^{*} p<0.1, * * p<0.05, * * * p<0.01$.

Nota: Entre paréntesis se muestra el rango del valor de la prueba AUDIT-OMS. Prueba $t$ de diferencia de medias.

Fuente: Elaboración propia con datos de la ENA, 2011. 
La ecuación que se desea estimar establece la asociación entre el consumo de alcohol, $A U D I T_{i}$, que influye sobre la probabilidad de que el adolescente trabaje, Dtrabajo $_{i}$. Existen también otras variables del contexto familiar, $X_{i}$, y otras características sociodemográficas, $W_{i}$, que influyen en la probabilidad de que el niño trabaje. La ecuación que se desea estimar es:

$$
\text { Dtrabajo }_{i}=\beta_{0}+\beta_{1} A U D I T_{i}+\beta_{2} X_{i}+\beta_{3} W_{i}+\varepsilon_{i}
$$

Por otra parte, también es posible explicar la relación de la participación laboral y el consumo de alcohol de forma inversa, dado que si el adolescente trabaja tendrá más posibilidades económicas para probar o incrementar el consumo de bebidas alcohólicas. Si existe una causalidad inversa, el término error, $\varepsilon_{i}$, contiene información que no se observa y que podría estar relacionada con un determinado consumo de alcohol.

$$
A U D I T_{i}=\beta_{0}^{\prime}+\beta_{1}^{\prime} \text { Dtrabajo }_{i}+\beta_{2}{ }_{2} X_{i}+\beta^{\prime}{ }_{3} W_{i}+\varepsilon^{\prime}{ }_{i}
$$

Maddala (2001) indica que si dos variables tienen una asociación en donde no se está seguro de la dirección de la causalidad, se estiman los coeficientes de las variables de interés. En este caso $\beta$, a partir de la estimación de la Ecuación 5, que consideramos como la relación directa, y de forma inversa la Ecuación 6, en donde la variable AUDIT se convierte en la variable dependiente, y la participación laboral en la independiente, cuyo coeficiente es $\beta^{\prime}{ }_{l}$. La intención es encontrar el intervalo en el cual oscila la asociación entre las variables. Este tipo de metodología se ha utilizado para analizar la discriminación por raza y género. En donde no se conoce el sentido de la causalidad, la conclusión es que, independientemente de la especificación, los coeficientes de las regresiones directas e inversas son parámetros de interés válidos (Conway y Roberts, 1983).

El Cuadro 2 muestra los resultados de la Ecuación 6, que mide el efecto inverso, es decir, el efecto de la participación laboral sobre la persistencia del consumo del alcohol medido por la variable AUDIT. La metodología para estimar la Ecuación 6 es la de mínimos cuadrados ordinarios (MCO), puesto que la variable dependiente es continua; para analizar la sensibilidad de este coeficiente se presentan cinco modelos. Los resultados muestran una asociación positiva pero significativa sólo en dos modelos. Se infiere que, si el adolescente trabaja, su consumo de alcohol medido por el valor del AUDIT aumenta entre 0.4681 y 0.822 unidades. El consumo alcohólico es mayor en los hombres y aumenta con la edad, pero no está relacionado con el sexo del jefe de familia ni con la compo- 
sición del hogar, el número de habitantes del hogar, el ingreso del hogar, y el acceso a los servicios médicos.

\section{Cuadro 2}

Regresión inversa

\begin{tabular}{|c|c|c|c|c|c|}
\hline & Modelo R1 & Modelo R2 & Modelo R3 & Modelo R4 & Modelo R5 \\
\hline $\begin{array}{l}\text { Dummy } \\
\text { de trabajo }\end{array}$ & $\begin{array}{l}0.4876 \\
(1.61)\end{array}$ & $\begin{array}{l}0.4681^{*} \\
(1.89)\end{array}$ & $\begin{array}{l}0.4939 \\
(1.64)\end{array}$ & $\begin{array}{l}0.485 \\
(1.6)\end{array}$ & $\begin{array}{l}0.822^{* *} \\
(2.34)\end{array}$ \\
\hline Género & $\begin{array}{l}0.868 * * * \\
(5.42)\end{array}$ & $\begin{array}{l}0.744 * * * \\
(5.82)\end{array}$ & $\begin{array}{l}0.862 * * * \\
(5.39)\end{array}$ & $\begin{array}{l}0.869 * * * \\
(5.43)\end{array}$ & $\begin{array}{l}0.805^{* * *} \\
(5.12)\end{array}$ \\
\hline Estudia & $\begin{array}{l}-1.15 * * * \\
(-3.15)\end{array}$ & $\begin{array}{l}-0.95 * * * \\
(-3.25)\end{array}$ & $\begin{array}{l}-1.17 * * * \\
(-3.24)\end{array}$ & $\begin{array}{l}-1.16^{* * *} \\
(-3.16)\end{array}$ & \\
\hline Edad & $\begin{array}{l}0.281 * * * \\
(4.52)\end{array}$ & $\begin{array}{l}0.328 * * * \\
(6.86)\end{array}$ & $\begin{array}{l}0.279 * * * \\
(4.54)\end{array}$ & $\begin{array}{l}0.281 * * * \\
(4.53)\end{array}$ & $\begin{array}{l}0.364 * * * \\
(6.63)\end{array}$ \\
\hline $\begin{array}{l}\text { Jefe de familia } \\
\text { hombre }\end{array}$ & $\begin{array}{l}-0.2449 \\
(-0.79)\end{array}$ & $\begin{array}{l}-0.2502 \\
(-1.11)\end{array}$ & $\begin{array}{l}-0.237 \\
(-0.77)\end{array}$ & & $\begin{array}{l}-0.3119 \\
(-1.00)\end{array}$ \\
\hline Hogar nuclear & $\begin{array}{l}-0.2113 \\
(-0.67)\end{array}$ & $\begin{array}{l}-0.1568 \\
(-0.68)\end{array}$ & $\begin{array}{l}-0.1965 \\
(-0.63)\end{array}$ & $\begin{array}{l}-0.419 * * \\
(-2.03)\end{array}$ & $\begin{array}{l}-0.1599 \\
(-0.51)\end{array}$ \\
\hline $\begin{array}{l}\text { Ingreso } \\
\text { del hogar }\end{array}$ & $\begin{array}{l}0.0471 \\
(0.56)\end{array}$ & & $\begin{array}{l}0.0472 \\
(0.56)\end{array}$ & $\begin{array}{l}0.046 \\
(0.55)\end{array}$ & $\begin{array}{l}0.0133 \\
(0.16)\end{array}$ \\
\hline $\begin{array}{l}\text { Habitantes } \\
\text { del hogar }\end{array}$ & $\begin{array}{l}0.019 \\
(0.52)\end{array}$ & $\begin{array}{l}0.0137 \\
(0.48)\end{array}$ & & $\begin{array}{l}0.019 \\
(0.51)\end{array}$ & $\begin{array}{l}0.0282 \\
(0.74)\end{array}$ \\
\hline $\begin{array}{l}\text { Densidad } \\
\text { poblacional }\end{array}$ & $\begin{array}{l}0.0001 \\
(1.21)\end{array}$ & $\begin{array}{l}0.0008 \\
(1.4)\end{array}$ & $\begin{array}{l}0.0001 \\
(1.26)\end{array}$ & $\begin{array}{l}0.0001 \\
(1.23)\end{array}$ & $\begin{array}{l}0.00009 \\
(1.03)\end{array}$ \\
\hline $\begin{array}{l}\text { Dummy } \\
\text { de seguro social } \\
\text { o privado }\end{array}$ & $\begin{array}{l}0.1411 \\
(0.62)\end{array}$ & $\begin{array}{l}0.1411 \\
(0.85)\end{array}$ & $\begin{array}{l}0.1405 \\
(0.62)\end{array}$ & $\begin{array}{l}0.142 \\
(0.63)\end{array}$ & $\begin{array}{l}-0.0233 \\
(-0.11)\end{array}$ \\
\hline Región norte & $\begin{array}{l}0.2377 \\
(1.32)\end{array}$ & $\begin{array}{l}0.1781 \\
(1.43)\end{array}$ & $\begin{array}{l}0.2302 \\
(1.28)\end{array}$ & $\begin{array}{r}0.237 \\
(1.32)\end{array}$ & $\begin{array}{l}0.2995 \\
(1.64)\end{array}$ \\
\hline $\begin{array}{l}\text { Región centro- } \\
\text { norte }\end{array}$ & $\begin{array}{l}0.5645 * * * \\
(2.83)\end{array}$ & $\begin{array}{l}0.541 * * * \\
(3.69)\end{array}$ & $\begin{array}{l}0.5635^{* * * *} \\
(2.83)\end{array}$ & $\begin{array}{l}0.565^{* * *} \\
(2.83)\end{array}$ & $\begin{array}{l}0.59 * * * \\
(2.9)\end{array}$ \\
\hline Región centro & $\begin{array}{l}0.7308 \\
(2.55) \\
\end{array}$ & $\begin{array}{l}0.691 * * * \\
(3.22)\end{array}$ & $\begin{array}{l}0.7248 * * \\
(2.52)\end{array}$ & $\begin{array}{l}0.725^{* *} \\
(2.53)\end{array}$ & $\begin{array}{l}0.745^{* *} \\
(2.53)\end{array}$ \\
\hline Observaciones & 2530 & 3751 & 2530 & 2530 & 2530 \\
\hline
\end{tabular}

$*_{p}<0.1, * * p<0.05, * * * p<0.01$.

Nota: Los estadísticos $t$ se muestran entre paréntesis.

Fuente: Elaboración propia con datos de la ENA, 2011. 
Para revisar si la estimación de los modelos del Cuadro 2, derivados de la Ecuación 6, ha sido realizada sin sesgo, se probará si existe correlación entre el término de error, $\varepsilon_{i}$, y la participación laboral. De la misma forma se probará si la Ecuación 5 estimada de forma directa presenta endogeneidad. Las habilidades socioemocionales no son observables. Como sugieren Berger, Milicic, Alcalay y Torretti (2014), un mayor desarrollo socioemocional, menor sintomatología depresiva y mayor satisfacción personal contribuyen a tener mejores logros educativos en los jóvenes y reducen la prevalencia de problemas conductuales.

Si existe endogeneidad entre el consumo de alcohol y la participación laboral, las estimaciones bajo MCO proporcionan coeficientes sesgados e inconsistentes (Bound, Jaeger y Baker, 1995). Una forma de corregir el sesgo es utilizando el método de variables instrumentales (Bound, Jaeger y Baker, 1995; Wooldridge, 2015). Para este propósito decidimos estimar la Ecuación 5 con el fin de identificar los factores relacionados con la participación laboral, entre ellos el consumo de alcohol de forma persistente. Debido a que la variable dependiente es binaria, se recomendaría utilizar un modelo probabilístico no lineal, conocido como modelo probit (Cameron y Trivedi, 2005), el cual supone una distribución normal en el error aleatorio, $\varepsilon_{i}$. Además, a partir de la ENA (2011) es posible incorporar variables relacionadas con la variable endógena AUDIT y que no están relacionadas de forma directa con la decisión de trabajar por parte del adolescente. Si bien podría pensarse que estos instrumentos también podrían influir en la participación laboral de los jóvenes, las pruebas de exogeneidad de los instrumentos permitirían conocer si existe asociación directa; de otra forma, la asociación entre los instrumentos y la participación laboral es a través del consumo persistente de alcohol.

Las personas cercanas al adolescente influyen en su comportamiento, en este caso, con respecto al consumo de bebidas alcohólicas (Pavis, Cunningham-Burley y Amos, 1997; Espada Sánchez, Pereira y García-Fernández, 2008; Salamó, Gras Pérez y Font-Mayolas, 2010). A partir de la encuesta ENA (2011) podemos conocer si el mejor amigo del menor consume drogas; la influencia de ese comportamiento incrementaría el consumo del adolescente y, de forma indirecta, afectaría la probabilidad de trabajar. Un estado de ánimo negativo de los individuos también influye en el consumo de alcohol, ya que las emociones negativas los impulsan a tomar bebidas alcohólicas con objeto de aliviar el sentimiento o dolor (Peirce, Frone, Russell y Cooper, 1994; Pavis, Cunningham-Burley y Amos, 1997). Por tanto, se añaden tres variables como instrumentos que indican el estado de ánimo del menor: la frecuencia con la que el adolescente se siente sin esperanza, la 
frecuencia con la que el adolescente se siente deprimido, y la frecuencia con la que al adolescente le cuesta mucho esfuerzo realizar sus actividades. Estos tres instrumentos nos permitirían identificar si existe alguna condición interna en los jóvenes que los haga más propensos a consumir alcohol y, por ende, a insertarse en el mercado laboral a una edad temprana. Además, estas tres variables son categóricas y toman valores entre cero y cuatro: el cero se asigna si los adolescentes nunca han tenido estos síntomas; el uno, si casi nunca se han sentido así; el dos, si la frecuencia es a veces; el tres, si la frecuencia es casi siempre; y el cuatro, si el adolescente siempre se siente de esa manera.

\section{Resultados}

A partir de la estrategia empírica se estimará la Ecuación 5 en dos etapas, de tal forma que, en la primera etapa, expresada en la Ecuación 7, se valoran diferentes combinaciones de los instrumentos, $Z_{j}$, donde en $j$ se incluye si el mejor amigo consume drogas, si se siente deprimido, sin esperanza, o si el joven requiere mucho esfuerzo para realizar sus actividades:

$$
A U D I T_{i}=\gamma_{1}+\gamma_{2} Z_{j}+v_{i}
$$

Las diferentes especificaciones de los instrumentos permitirán evaluar la validez, debilidad y sensibilidad de los instrumentos. Se incluyen modelos exactamente identificados, considerando sólo un instrumento, y modelos sobreidentificados, incorporando más de uno.

La segunda etapa consiste en estimar la Ecuación 5, pero añadiendo el valor predicho de la variable AUDIT obtenido en la primera etapa. Para este propósito, se analizan modelos incorporando distintas variables de control para examinar la sensibilidad y robustez de la relación entre el consumo persistente de alcohol y la probabilidad de participar en el mercado laboral. La ecuación a estimar en la segunda etapa es:

$$
\text { Dtrabajo }_{i}=\beta_{0}+\beta_{1} A \widehat{U D I} T_{i}+\beta_{2} X_{i}+\beta_{3} W_{i}+\varepsilon_{i}
$$

\subsection{Resultados de la primera etapa}

Murray (2006) subraya el cuidado que se debe tener al usar variables instrumentales, ya que no sólo hay que encontrar instrumentos que sean válidos, 
sino también deben ser robustos; de otra forma, el estimador obtenido sería sesgado e inconsistente, aún más que el arrojado por el modelo probit sin variables instrumentales. La primera etapa de la estimación proporciona los elementos para evaluar si los instrumentos propuestos son relevantes, no débiles y válidos. En la primera parte del Cuadro 3 se presentan los coeficientes obtenidos de estimar un modelo por MCO de cada instrumento y la variable AUDIT. La prueba de Wu-Hausman se presenta para mostrar que la variable AUDIT es endógena, por lo que la estimación con variables instrumentales se prefiere a la estimación sin corrección. En la segunda parte del Cuadro 3 se presentan distintas especificaciones que combinan más de un instrumento, es decir, son modelos sobreidentificados.

La significancia estadística de los instrumentos en las distintas especificaciones indica que la influencia de los pares y las habilidades socioemocionales de los jóvenes son relevantes pues están correlacionadas con el consumo persistente de alcohol, medido por AUDIT. Esto es, si el mejor amigo consume droga, se asocia a un mayor consumo de alcohol: 2.95; si está deprimido: 0.776; si no tiene esperanza: 0.786; y si requiere mucho esfuerzo para realizar sus actividades: 0.392. Las distintas especificaciones con sobreidentificación muestran que los coeficientes se mantienen positivos y significativos, con excepción del instrumento "requiere mucho esfuerzo", ya que está asociado a un mayor consumo, pero si se combina con los otros tres instrumentos, la asociación reduce el consumo persistente de alcohol. Siguiendo a Staiger y Stock (1997), podemos inferir que tres de los cuatro instrumentos utilizados no son débiles (excepto el instrumento "requiere mucho esfuerzo"), ya que, en cada una de las estimaciones de la primera etapa, tanto en los modelos exactamente identificados y sobreidentificados, el estadístico $\mathrm{F}$ es mayor a 10, por lo que superan el valor crítico. El valor del estadístico F más alto se encuentra en el modelo 1.1, en donde únicamente se incluye como instrumento si el mejor amigo consume drogas. Otra forma de revisar la relevancia del instrumento es comparando la R-cuadrada parcial de Shea (Shea partial R2), en donde es notable que el modelo 1.1 es el que tiene el mayor valor en comparación con los otros instrumentos exactamente identificados, aunque cuando se compara con los modelos sobreidentificados el valor aumenta conforme se incluyen más instrumentos.

De acuerdo con Sargan (1958), se prueba la validez de los instrumentos revisando si éstos son exógenos, para lo que se requiere más de un instrumento; es decir, sólo se aplica en los modelos sobreidentificados. En la columna se muestran los resultados de la prueba de Sargan-Hansen, así como la significancia de la prueba conjunta al incluir los instrumentos, prueba F y 
la correlación parcial de incorporar los instrumentos en cada especificación, medida por la R-cuadrada parcial de Shea (Shea partial R2). Los resultados indican que las especificaciones son correctas en los modelos; la prueba de Sargan-Hansen muestra que la hipótesis nula de que los instrumentos son exógenos no se rechaza, por lo que se infiere que las combinaciones propuestas implican instrumentos válidos. Con fines de revisar la robustez del coeficiente de AUDIT ante las diferentes especificaciones, en la segunda etapa se utilizarán estos modelos para estimar el efecto del consumo persistente de alcohol sobre la probabilidad de que el adolescente trabaje.

\subsection{Resultados de la segunda etapa}

En la segunda etapa se incluye la variable predicha del AUDIT bajo distintas especificaciones de los instrumentos y diferentes combinaciones de las variables exógenas relacionadas con la participación laboral de los adolescentes; se incluyen variables sociodemográficas, como la edad y el sexo de éstos. También se incluyen variables económicas, como la asistencia escolar, el ingreso familiar en salarios mínimos y el número de habitantes en el hogar, ya que mientras más personas vivan en el hogar, mayor será el ingreso familiar necesario para poder subsistir y, por ende, mayor será la probabilidad de que el menor trabaje. El trabajo de los jóvenes menores de edad es más común en las zonas rurales (UCW, 2012); por eso se añade la densidad poblacional por estado como proxy del tamaño de la localidad. Por último, se añaden variables relacionadas con el hogar, como el género del jefe de familia y si el adolescente vive en un hogar nuclear, compuesto por ambos padres, o si vive sólo con alguno de los dos. La mayoría de los jóvenes que trabajan lo hacen en modalidades informales (UCW, 2012), por lo que se agrega la variable de seguro médico, que toma el valor de uno si el menor cuenta con éste, y cero si no lo tiene.

En el Cuadro 4 se muestran los factores relacionados con la probabilidad de que el adolescente trabaje. El primer modelo es el efecto marginal de un modelo probit sin instrumento, mientras que los modelos 2.1-2.4 se obtienen de incorporar en la primera etapa cada uno de los instrumentos, modelos 1.1-1.4 respectivamente. Dados los resultados de la prueba de Wu-Hausman, mostrados en el Cuadro 3, la variable AUDIT es endógena, por lo que el coeficiente está sesgado. 


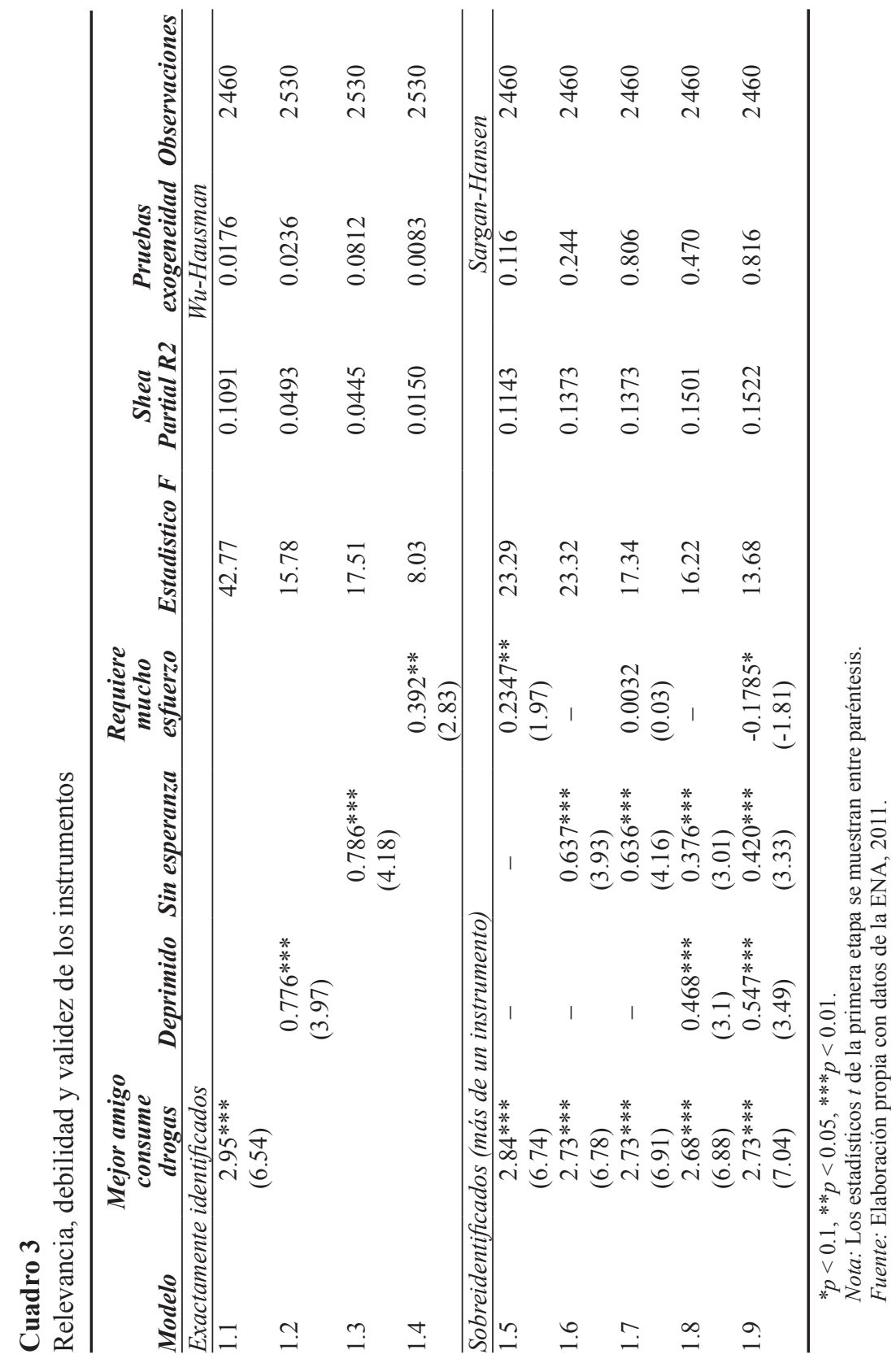




\section{Cuadro 4}

Efectos marginales sobre la probabilidad de que los adolescentes trabajen

\begin{tabular}{|c|c|c|c|c|c|}
\hline \multirow[b]{3}{*}{ Instrumento } & \multicolumn{5}{|c|}{ Modelo } \\
\hline & 2.0 & 2.1 & 2.2 & 2.3 & 2.4 \\
\hline & $\begin{array}{c}\text { Sin } \\
\text { instrumento } \\
d y / d x\end{array}$ & $\begin{array}{c}\text { Mejor amigo } \\
d y / d x\end{array}$ & $\begin{array}{l}\text { Deprimido } \\
\qquad d y / d x\end{array}$ & $\begin{array}{c}\text { Sin } \\
\text { esperanza } \\
d y / d x\end{array}$ & $\begin{array}{c}\text { Requiere } \\
\text { mucho } \\
\text { esfuerzo } \\
d y / d x\end{array}$ \\
\hline \multirow[t]{2}{*}{ AUDIT } & 0.0035 & $0.022 * *$ & $0.0304 * *$ & 0.0207 & $0.0486^{*}$ \\
\hline & $(1.26)$ & $(2.42)$ & $(2.08)$ & $(1.32)$ & $(1.88)$ \\
\hline \multirow[t]{2}{*}{ Género } & $0.2293^{* * *}$ & $0.2054 * * *$ & $0.2056^{* * *}$ & $0.214 * * *$ & $0.1874 * * *$ \\
\hline & (10.97) & $(9.49)$ & $(8.82)$ & $(8.92)$ & $(6.29)$ \\
\hline \multirow{2}{*}{$\begin{array}{l}\text { Dummy } \\
\text { estudia }\end{array}$} & $-0.252 * * *$ & $-0.2153^{* * *}$ & $-0.1991 * * *$ & $-0.2178 * * *$ & $-0.1671 * * *$ \\
\hline & $(-6.86)$ & $(-5.46)$ & $(-4.88)$ & $(-5.03)$ & $(-3.09)$ \\
\hline \multirow[t]{2}{*}{ Edad } & $0.0514 * * *$ & $0.0438 * * *$ & $0.0437 * * *$ & $0.0463^{* * *}$ & $0.0379 * * *$ \\
\hline & $(6.54)$ & $(5.01)$ & $(4.72)$ & $(4.74)$ & $(3.2)$ \\
\hline \multirow{2}{*}{$\begin{array}{l}\text { Dummy jefe } \\
\text { familia }\end{array}$} & -0.0031 & 0.0036 & 0.0019 & 0.001 & 0.007 \\
\hline & $(-0.07)$ & $(0.09)$ & $(0.05)$ & $(0.02)$ & $(0.17)$ \\
\hline \multirow{2}{*}{$\begin{array}{l}\text { Dummy hogar } \\
\text { nuclear }\end{array}$} & 0.0261 & 0.0245 & 0.0313 & 0.0282 & 0.0358 \\
\hline & $(0.63)$ & $(0.61)$ & $(0.76)$ & $(0.68)$ & $(0.87)$ \\
\hline \multirow{2}{*}{$\begin{array}{l}\text { Ingreso } \\
\text { del hogar }\end{array}$} & $-0.0253^{* * *}$ & $-0.026^{* * *}$ & $-0.0269 * * *$ & $-0.0268 * * *$ & $-0.0283^{* * *}$ \\
\hline & $(-2.60)$ & $(-2.64)$ & $(-2.78)$ & $(-2.78)$ & $(-2.9)$ \\
\hline \multirow{2}{*}{$\begin{array}{l}\text { Habitantes } \\
\text { del hogar }\end{array}$} & 0.0021 & 0.0019 & 0.0015 & 0.0017 & 0.0011 \\
\hline & $(1.28)$ & $(1.16)$ & $(0.91)$ & (1.04) & $(0.67)$ \\
\hline \multirow{2}{*}{$\begin{array}{l}\text { Densidad } \\
\text { poblacional }\end{array}$} & $-0.000016^{*}$ & $-0.000016^{*}$ & $-0.000021^{* *}$ & $-0.000019 * *$ & $-0.000023^{* *}$ \\
\hline & $(-1.88)$ & $(-1.84)$ & $(-2.41)$ & $(-2.19)$ & $(-2.49)$ \\
\hline \multirow{2}{*}{$\begin{array}{l}\text { Seguro social } \\
\text { o privado }\end{array}$} & -0.0173 & -0.0246 & -0.0192 & -0.0199 & -0.0211 \\
\hline & $(-0.70)$ & $(-0.97)$ & $(-0.77)$ & $(-0.80)$ & $(-0.84)$ \\
\hline Región & Sí & Sí & Sí & Sí & Sí \\
\hline Observaciones & 2530 & 2460 & 2530 & 2530 & 2530 \\
\hline
\end{tabular}

${ }^{*} p<0.1,{ }^{* *} p<0.05,{ }^{* * *} p<0.01$.

Nota: Variable dependiente: dummy igual a $1 \mathrm{si}$ el adolescente trabaja; igual a 0 si no lo hace. Los estadísticos $z$ se muestran entre paréntesis.

Fuente: Elaboración propia con datos de la ENA, 2011.

Además, al comparar el coeficiente del modelo 2.0 con los coeficientes de AUDIT mostrados en el Cuadro 2 (obtenido de la regresión inversa), encontramos que los coeficientes tienen signo positivo, lo que indica una 
asociación positiva entre el consumo persistente de alcohol y la participación laboral de los adolescentes. Cuando no se corrige por variables instrumentales, los coeficientes del AUDIT muestran amplia variabilidad ante las distintas especificaciones de las variables de control, y en la mayoría de los modelos resulta estadísticamente no significativa.

Cuando se corrige por variables instrumentales, con modelos exactamente identificados, la magnitud de los coeficientes de AUDIT aumenta, por lo que inferimos que el sesgo por autoselección era más fuerte. Así también, deducimos que la relación oscila en un menor rango de valores, y sólo con excepción del modelo en donde el adolescente se siente sin esperanza, los coeficientes del AUDIT son significativos, por lo que consideramos que el consumo persistente de alcohol incrementa la probabilidad de que los adolescentes trabajen. Dependiendo del instrumento la magnitud varía: si el mejor amigo consume drogas, la probabilidad de que el adolescente trabaje se incrementa en 2.2 puntos porcentuales (pp); si se siente deprimido, la probabilidad aumenta en $3.04 \mathrm{pp}$; si el adolescente refiere que realiza sus actividades con mucho esfuerzo, la probabilidad de que trabaje aumenta en $4.86 \mathrm{pp}$, con una significancia estadística de al menos $10 \%$. Si bien parecen contraintuitivos los resultados, hay que considerar que el efecto que se está identificando es la incidencia de un alto consumo de alcohol sobre la probabilidad de trabajar. Con respecto a las variables de control, se encuentra que es más probable que los hombres se inserten al mercado laboral en comparación con las mujeres, entre 18.74 pp y 22.93 pp; así también, la probabilidad de trabajar aumenta con la edad entre 3.79 pp y 5.14 pp. El coeficiente del ingreso del hogar es negativo y resulta significativo al $1 \%$ en todas las estimaciones presentadas. Es decir, si aumenta el ingreso del hogar, la probabilidad de que el menor trabaje disminuye en un rango de entre 2.5 y 2.83 pp; así también la probabilidad de trabajar se reduce si la localidad es de más de un millón de habitantes. No se encuentra un efecto estadísticamente significativo entre el número de integrantes en el hogar; tampoco si el adolescente tiene acceso a servicio médico sobre la probabilidad de que el mismo trabaje.

El efecto marginal de la estimación sólo para los coeficientes del AUDIT se muestra en el Cuadro 5, con el fin de conocer la sensibilidad de la relación entre el consumo persistente del alcohol sobre la probabilidad de trabajar. En la primera parte se presentan modelos excluyendo algunas de las variables de control en los modelos exactamente identificados en la primera etapa. En la segunda parte del Cuadro 5 se añaden los coeficientes que se obtienen al incorporar modelos sobreidentificados, a partir de las combinaciones de los instrumentos realizados en la primera etapa de la estimación. 


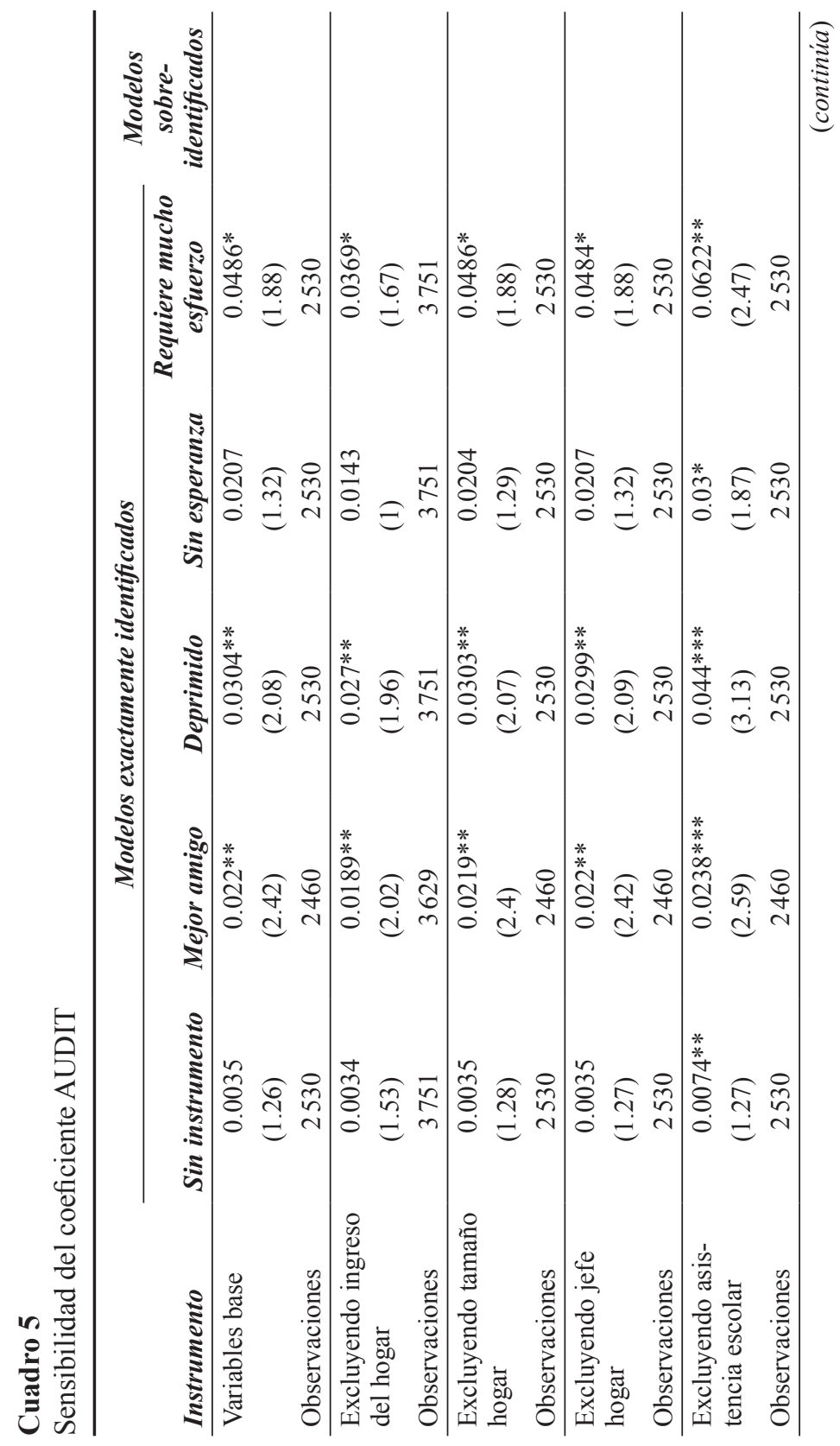




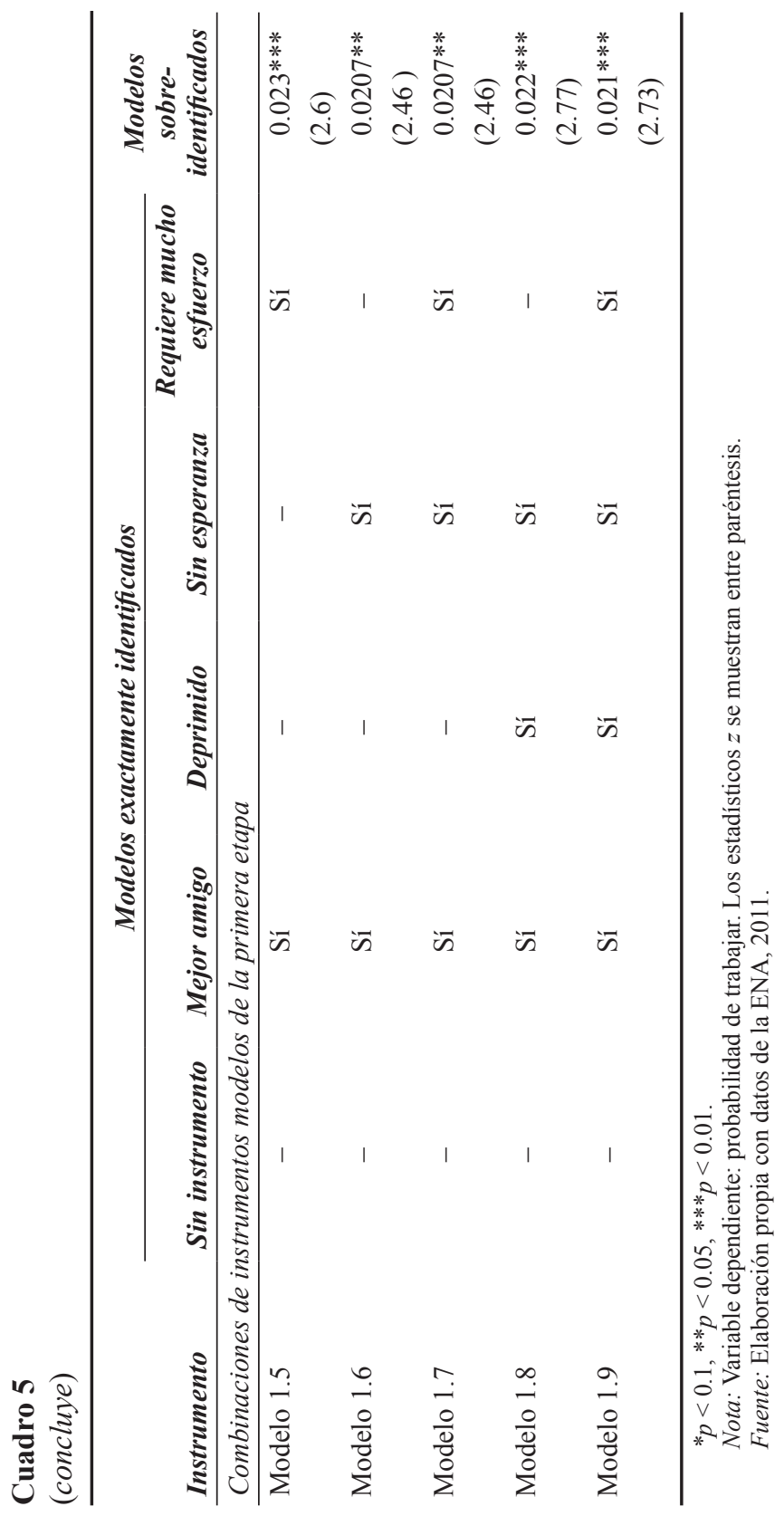


El Cuadro 5 permite comparar la sensibilidad de los coeficientes del AUDIT ante las diferentes especificaciones y estima que el efecto tiene signo positivo y es significativo, con excepción del modelo sin corregir y el modelo en donde el adolescente menciona que se siente sin esperanza. Cuando se excluye la variable de asistencia escolar, todos los coeficientes se vuelven significativos y aumentan su magnitud. Esto podría implicar un sesgo de habilidad en el modelo sin corregir: si los jóvenes menos hábiles prefieren dejar la escuela, tendrán mayor probabilidad de entrar al mercado laboral. En ese caso, el coeficiente estaría sobreestimado si no se incluye la variable de asistencia escolar. Por otra parte, si los jóvenes que eligen consumir alcohol de forma persistente y aquellos que deciden trabajar son similares, podríamos inferir que el sesgo es por autoselección, o bien, porque los jóvenes subreportan la frecuencia en el consumo del alcohol, por lo que los coeficientes sin corregir estarían subestimados.

En resumen, por los coeficientes obtenidos de los modelos, con instrumentos exactamente identificados y sobreidentificados, inferimos que la variable AUDIT tiene signo positivo, es significativa y robusta ante cualquier combinación de instrumentos. En los modelos en donde se combinan los instrumentos parece haber coeficientes con menor sensibilidad ante las especificaciones; por tanto, se infiere que el consumo persistente de alcohol incide en la participación laboral adolescente en un rango entre 2.07 pp y 2.34 pp.

\section{Conclusiones}

La evidencia empírica obtenida en este artículo demuestra una relación directa y significativa entre el consumo de bebidas alcohólicas y el trabajo adolescente en México; es decir, entre mayor sea el consumo de alcohol del joven, aumentará su probabilidad de que ingrese al mercado laboral. En cada uno de los modelos presentados la variable que mide la intensidad del consumo de alcohol (variable AUDIT) resultó significativa con cada uno de los instrumentos utilizados, con un nivel de confiabilidad de al menos 90\%. También se encuentra que los hombres tienen más probabilidad de trabajar en comparación con las mujeres. Además, un incremento en el ingreso del hogar reduce la probabilidad de que los menores de edad trabajen.

La información acerca del estado de ánimo del adolescente, así como la del comportamiento de las personas con las cuales convive, pueden usarse como instrumentos para medir su consumo de alcohol, ya que cumplen con las condiciones necesarias para identificar instrumentos robustos. A partir de las pruebas de validez de instrumentos se infiere que la combinación de 
éstos permite tener una mayor robustez en la relación entre el consumo persistente de alcohol y la participación laboral de los adolescentes.

Una limitación de la estimación es que no tenemos variables de control como la escolaridad del jefe de familia (UCW, 2012), si el hogar recibe remesas (Alcaraz, Chiquiar y Salcedo, 2012), o si el hogar obtiene transferencias de algún programa gubernamental, lo que reduciría la probabilidad de trabajar del menor (Skoufias, Parker, Behrman y Pessino, 2001; Schultz, 2004; Ranzani y Rosati, 2014). Sin embargo, la limitante más importante es que en la encuesta utilizada no se puede identificar el trabajo peligroso y el no peligroso, lo cual es de suma importancia debido a que la severidad en el consumo del alcohol es mayor entre los jóvenes que trabajan, ya que es probable que estemos identificando a aquellos con mayor adicción y dispuestos a realizar trabajos riesgosos y peligrosos.

Por último, se recomienda seguir impulsando proyectos informativos que ahonden en la problemática de los niños, adolescentes y jóvenes. Se sugiere continuar el monitoreo de la situación laboral de los jóvenes a través del Módulo de Trabajo Infantil (MTI) de la Encuesta Nacional de Ocupación y Empleo (ENOE); o bien, enriquecer la Encuesta Nacional de Adicciones, incorporando reactivos relacionados con aspectos laborales específicos que permitan identificar la evolución de las condiciones al respecto y plantear programas que impulsen el desarrollo de los jóvenes.

\section{Bibliografía}

Alcaraz, C., Chiquiar, D. y Salcedo, A. (2012). Remittances, schooling, and child labor in Mexico. Journal of Development Economics, 97(1), 156165. Recuperado de https://www.sciencedirect.com/science/article/ abs/pii/S0304387810001318?via\%3Dihub

Babor, T., Higgins-Biddle, J., Saunders, J. y Monteiro, M. (2001). Cuestionario de identificación de los trastornos debidos al consumo de alcohol. Ginebra: Organización Mundial de la Salud. Recuperado de https://www.who.int/substance_abuse/activities/en/AUDITmanual Spanish.pdf

Basu, K. y Hoang Van, P. (1998). The economics of child labor. The American Economic Review, 88(3), 412-427. Recuperado de http://www. jstor.org/stable/116842

Becker, G. y Murphy, K. (1988). A theory of rational addiction. Journal of Political Economy, 96(4), 675-700. Recuperado de https://www. journals.uchicago.edu/doi/10.1086/261558 
Beegle, K., Dehejia, R. y Gatti, R. (2009). Why should we care about child labor? The education, labor market, and health consequences of child labor. Journal of Human Resources, 44(4), 871-889. Recuperado de http://jhr.uwpress.org/content/44/4/871.short

Berger, C., Milicic, N., Alcalay, L. y Torretti, A. (2014). Programa para el bienestar y aprendizaje socioemocional en estudiantes de tercero y cuarto grado: descripción y evaluación de impacto. Revista Latinoamericana de Psicología, 46(3), 169-177. Recuperado de https://www. sciencedirect.com/science/article/pii/S0120053414700202?via\%3 Dihub

Bound, J., Jaeger, D. y Baker, R. (1995). Problems with instrumental variables estimation when the correlation between the instruments and the endogenous explanatory variable is weak. Journal of the American Statistical Association, 90(430), 443-450. Recuperado de https://www. tandfonline.com/doi/abs/10.1080/01621459.1995.10476536

Cahuc, P. y Zylberberg, A. (2004). Labor economics, MIT Press.

Cameron, A. y Trivedi, P. (2005). Microeconometrics: Methods and applications. Cambridge University Press.

Conway, D. y Roberts, H. (1983). Reverse regression, fairness, and employment discrimination. Journal of Business and Economic Statistics, 1(1), 75-85. Recuperado de https://amstat.tandfonline.com/doi/ abs/10.1080/07350015.1983.10509326\#.XoVVNupKjIU

De Janvry, A., Finan, F. y Sadoulet, E. (2004). Can conditional cash transfers serve as safety nets to keep children at school and out of the labor market? Berkeley: University of California, Department of Agricultural and Resource Economics. Recuperado de https://escholarship.org/ uc/item $/ 5 \mathrm{fp} 0 \mathrm{~g} 5 \mathrm{p} 2$

Edmonds, E. (2007). Child labor. (Documento de Trabajo, núm. 2626). Bonn, Alemania: IZA Institute of Labor Economics. Recuperado de http://nbn-resolving.de/urn:nbn:de:101:1-20080422146

ENA. (2011). Encuesta Nacional de Adicciones 2011: reporte de drogas. Ciudad de México: Instituto Nacional de Psiquiatría Ramón de la Fuente Muñiz / Instituto Nacional de Salud Pública / Secretaría de Salud. Recuperado de http://www.conadic.salud.gob.mx/pdfs/ENA_2011_ DROGAS_ILICITAS_.pdf

Encodat. (2016). Encuesta Nacional de Consumo de Drogas, Alcohol y Tabaco 2016-2017: reporte de alcohol. Ciudad de México: Instituto Nacional de Psiquiatría Ramón de la Fuente Muñiz / Instituto Nacional de Salud Pública / Secretaría de Salud. Recuperado de https://www.insp. $\mathrm{mx} /$ avisos/4585-encodat-2016.html 
Espada Sánchez, J., Pereira, J. y García-Fernández, J. (2008). Influencia de los modelos sociales en el consumo de alcohol de los adolescentes. Psicothema, 20(4), 531-537. Recuperado de www.unioviedo.net/reunido/ index.php/PST/article/download/8693/8557

Forastieri, V. (2002). Children at work: Health and safety risks. International Labour Organization. Recuperado de https://www.ilo.org/global/ topics/safety-and-health-at-work/resources-library/publications/ WCMS_235332/lang--en/index.htm

Grootaert, C. y Kanbur, R. (1994). Child labour: A review. (Documento de Trabajo, núm. 1454). The World Bank. Recuperado de http://gdsnet. org/Child-Labor-aReview.PDF

Guarcello, L., Lyon, S. y Valdivia, C. (2016). Adolescents in hazardous work: Child labour among children aged 15-17 years. (Documento de Trabajo). Understanding Children's Work (UCW). Recuperado de http:// www.ucw-project.org/attachment/23052017377Hazardous_work_ adolescents_in_CL_july2016.pdf

Guzmán, F., Pedrão, L., Rodríguez, L., López, S. y Esparza, S. (2007). Trastornos por consumo de alcohol (AUDIT) en adolescentes y jóvenes marginales de bandas juveniles de México. Escola Anna Nery. Revista de Enfermagem, 11(4), 1-11. Recuperado de http://www.scielo. br/scielo.php?script $=$ sci_arttext\&pid=S1414-81452007000400009\& lng=es\&tlng=es

Harris, M. y Fennell, M. (1988). A multivariate model of job stress and alcohol consumption. The Sociological Quarterly, 29(3), 391-406. Recuperado de http://www.jstor.org/stable/4121498

Hawkins, D., Catalano, R. y Miller, Y. (1992). Risk and protective factors for alcohol and other drug problems in adolescence and early adulthood: Implications for substance abuse prevention. Psychological Bulletin, 112(1), 64-105. Recuperado de https://psycnet.apa.org/ doiLanding?doi=10.1037\%2F0033-2909.112.1.64

Heady, C. (2003). The effect of child labor on learning achievement. World Development, 31(2), 385-398. Recuperado de https://www.science direct.com/science/article/abs/pii/S0305750X02001869?via\%3Dihub

Ilahi, N., Orazem, P. y Sedlacek, G. (2000). The implications of child labor for adult wages, income and poverty: Retrospective evidence from Brazil. The World Bank. Recuperado de https://www.researchgate. net/publication/247904326_The_Implications_of_Child_Labor_for_ Adult_Wages_Income_and_Poverty_Retrospective_Evidence_from_ Brazil 
International Labour Organization (ILO). (2013). Global child labour trends 2008 to 2012. International Labour Office, International Programme on the Elimination of Child Labour. Recuperado de https:// www.ilo.org/ipec/informationresources/WCMS_IPEC_PUB_23015/ lang--en/index.htm

Kandel, D. y Yamaguchi, K. (1987). Job mobility and drug use: An event history analysis. American Journal of Sociology, 92(4), 836-878. Recuperado de https://www.journals.uchicago.edu/doi/10.1086/228585

Kassouf, A., McKee, M. y Mossialos, E. (2001). Early entrance to the job market and its effect on adult health: Evidence from Brazil. Health Policy and Planning, 16(1), 21-28. Recuperado de https://academic.oup. com/heapol/article/16/1/21/617048

Keng, S. (1998). The demand for health, alcohol abuse, and labor market outcomes: A longitudinal study. Retrospective Theses and Dissertations, 11935. Iowa State University. Recuperado de https://lib.dr. iastate.edu/rtd/11935

Keng, S. y Huffman, W. (2007). Binge drinking and labor market success: A longitudinal study on young people. Journal of Population Economics, 20(1), 35-54. Recuperado de https://ideas.repec.org/a/spr/jopoec/ v20y2007ilp35-54.html

Maddala, G. (2001). Introduction to econometrics. Chichester, Reino Unido: John Willey and Sons.

Módulo de Trabajo Infantil (MTI). (2017). Principales resultados. Encuesta Nacional de Ocupación y Empleo. Cuarto trimestre. Instituto Nacional de Estadística y Geografía, INEGI. Recuperado de https://www.inegi. org.mx/contenidos/programas/mti/2017/doc/mti2017_resultados.pdf

Mullahy, J. y Sindelar, J. (1993). Alcoholism, work, and income. Journal of Labor Economics, 11(3), 494-520. Recuperado de https://www. journals.uchicago.edu/doi/10.1086/298305

Murray, M. (2006). Avoiding invalid instruments and coping with weak instruments. Journal of Economic Perspectives, 20(4), 111-132. Recuperado de https://www.aeaweb.org/articles?id=10.1257/jep.20.4.111

OECD. (2015). Tackling harmful alcohol use economics and public health policy: Economics and public health policy. OECD Publishing. Recuperado de https://www.oecd.org/health/tackling-harmful-alcohol-use9789264181069-en.htm

Pavis, S., Cunningham-Burley, S. y Amos, A. (1997). Alcohol consumption and young people: Exploring meaning and social context. Health Education Research, 12(3), 311-322. Recuperado de https://academic.oup. com/her/article/12/3/311/683115 
Peirce, R., Frone, M., Russell, M. y Cooper, M. (1994). Relationship of financial strain and psychosocial resources to alcohol use and abuse: The mediating role of negative affect and drinking motives. Journal of Health and Social Behavior, 35(4), 291-308. Recuperado de http:// www.jstor.org/stable/2137211

Psacharopoulos, G. (1997). Child labor versus educational attainment some evidence from Latin America. Journal of Population Economics, 10(4), 377-386. Recuperado de https://link.springer.com/article/10.1007 $\% 2 \mathrm{Fs} 001480050049$

Ranzani, M. y Rosati, F. (2014). Revisiting the impact of Oportunidades on children's activity in Mexico. (Documento de Trabajo). Understanding Children's Work (UCW). Recuperado de http://www. ucw-project.org/attachment/UCW_Oportunidades_impact_child_ activity20150219_154855.pdf

Ray, R. (2002). The determinants of child labour and child schooling in Ghana. Journal of African Economies, 11(4), 561-590. Recuperado de https://academic.oup.com/jae/article-abstract/11/4/561/750238? redirectedFrom=fulltext

Reinert, F. y Allen, J. (2002). The alcohol use disorders identification test (AUDIT): A review of recent research. Alcoholism: Clinical and Experimental Research, 26(2), 272-279. Recuperado de https://online library.wiley.com/doi/abs/10.1111/j.1530-0277.2002.tb02534.x

Salamó, A., Gras Pérez, M. y Font-Mayolas, S. (2010). Patrones de consumo de alcohol en la adolescencia. Psicothema, 22(2), 189-295. Recuperado de http://www.psicothema.com/psicothema.asp?id=3714

San José, B., van de Mheen, H., van Oers, J., Mackenbach, J. y Garretsen, H. (2000). Adverse working conditions and alcohol use in men and women. Alcoholism: Clinical and Experimental Research, 24(8), 1207-1213. Recuperado de https://onlinelibrary.wiley.com/doi/abs/ 10.1111/j.1530-0277.2000.tb02085.x

Sargan, J. (1958). The estimation of economic relationships using instrumental variables. Econometrica. Journal of the Econometric Society, 26(3), 393-415. Recuperado de https://www.jstor.org/stable/1907619

Schultz, T. (2004). School subsidies for the poor: Evaluating the Mexican Progresa poverty program. Journal of Development Economics, 74(1), 199-250. Recuperado de https://www.sciencedirect.com/science/ article/abs/pii/S0304387803001858?via\%3Dihub

Selvanathan, E. (1991). Cross-country alcohol consumption comparison: An application of the Rotterdam demand system. Journal Applied Eco- 
nomics, 23(10), 1613-1622. Recuperado de https://www.tandfonline. com/doi/abs/10.1080/00036849100000126

Selvanathan, E. y Selvanathan, S. (2004). Economic and demographic factors in Australian alcohol demand. Journal Applied Economics, 36(21), 2405-2417. Recuperado de https://www.tandfonline.com/doi/ abs/10.1080/0003684042000280346

Skoufias, E., Parker, S., Behrman, J. y Pessino, C. (2001). Conditional cash transfers and their impact on child work and schooling: Evidence from the Progresa program in Mexico. Economía, 2(1), 45-96. Recuperado de https://www.jstor.org/stable/20065413

Staiger, D. y Stock, J. (1997). Instrumental variables regressions with weak instruments. (Documento de Trabajo, núm. 151). Massachusetts: National Bureau of Economic Research. Recuperado de https://www.nber. org/papers/t0151

Telumbre-Terrero, J. y Sánchez-Jaimes, B. (2015). Consumo de alcohol en adolescentes del estado de Guerrero, México. Health and Addictions, 15(1), 79-86. Recuperado de https://ojs.haaj.org/index.php/haaj/article/ view/217

Understanding Children's Work (UCW). (2012). La experiencia mexicana en la reducción del trabajo infantil: evidencia empírica y lecciones políticas. Roma, Italia: Programa Entendiendo el Trabajo Infantil. Recuperado de http://www.ucw-project.org/

Wooldridge, J. (2015). Introductory econometrics: A modern approach. Nelson Education. Recuperado de https:/economics.ut.ac.ir/documents/ 3030266/14100645/Jeffrey_M._Wooldridge_Introductory_Econometrics_A_Modern_Approach_2012.pdf

\section{Acerca de los autores}

Juan Francisco Suárez Martínez es profesor de asignatura en el Departamento de Economía de la Universidad de Monterrey. Es licenciado en Matemáticas, maestro en Economía Industrial y candidato a doctor en Ciencias Económicas por la Universidad Autónoma de Nuevo León. Sus campos de estudio son: economía laboral y organización industrial, desarrollo económico regional, informalidad y econometría. Participó en el estudio del trabajo infantil y consumo de alcohol en México, impulsado por Understanding Children's Work (UCW), iniciativa conjunta de Unicef, Banco Mundial y Organización Internacional del Trabajo. Ha realizado estancias de investigación en la Universidad de Guadalajara y en el Banco de México, 
donde participó en investigaciones dedicadas a temas de informalidad y de salarios, respectivamente. ORCID: https://orcid.org/0000-0002-6256-0551

Cinthya G. Caamal Olvera obtuvo el doctorado y la maestría en Economía por la University of Essex, Inglaterra, y es licenciada en Economía por la Universidad Autónoma de Nuevo León (UANL). Actualmente es profesora de tiempo completo en la Facultad de Economía de la UANL, institución donde también coordinó los estudios sobre trabajo infantil en México -realizados por estudiantes-, promovidos por Understanding Children's Work (UCW), iniciativa conjunta de Unicef, Banco Mundial y Organización Internacional del Trabajo. Pertenece al Sistema Nacional de Investigadores del Conacyt, nivel I, y cuenta con reconocimiento al Perfil Deseable PRODEP, otorgado por la Secretaría de Educación. Ha publicado artículos en revistas académicas nacionales y extranjeras, en temas de desarrollo económico, economía laboral, economía de la educación, análisis de la pobreza, política fiscal, así como sobre cuestiones sectoriales y de seguridad social. ORCID https://orcid.org/0000-0003-0249-4027

Entre sus publicaciones destacan:

Carmona, N. y Caamal-Olvera, C. G. (2018). ¿Las transferencias federales han logrado reducir la desigualdad en las entidades federativas en México? EconoQuantum, 15(1), 31-51. Recuperado de http:// www.scielo.org.mx/scielo.php?script=sci_arttext\&pid=S1870-6622 2018000100031\&lng=es\&nrm=iso

Acosta, R. y Caamal-Olvera, C. G. (2017). Las remesas y la permanencia escolar en México. Migraciones Internacionales, 9(2), 85-111. Recuperado de https://migracionesinternacionales.colef.mx/index.php/ migracionesinternacionales/article/view/1333/1163

Caamal-Olvera, C. G. (2017). Decreasing returns to schooling in Mexico. Estudios Económicos, 32(1), 27-63. Recuperado de https://estudios economicos.colmex.mx/index.php/economicos/article/view/2/2

Recepción: 21 de junio de 2019. Aceptación: 18 de marzo de 2020. 
